\title{
Power Prediction of Multipath Components in Wireless MIMO Channels
}

\author{
Dmitriy Shutin, Gernot Kubin \\ Signal Processing and Speech Communication Laboratory \\ Graz University of Technology \\ Graz, Austria \\ dshutin@tugraz.at, gernot.kubin@tugraz.at
}

\begin{abstract}
Wireless systems are subject to fading - time variations of the receiving conditions caused by multipath propagation and transceiver movements. Prediction of fading allows to 'learn' the channel state information (CSI) in advance and adjust the transmission scheme as required based on the future values of CSI. In this contribution we propose a framework to handle predictions of general fast- and non-flat fading MIMO wireless channels. Unlike current approaches to predict channels by feeding sampled channel impulse response taps into the predictor, we first estimate multipath parameters, such as delay, Doppler frequencies, DoD/DoA, and design predictors for them. This step decreases the rate of variation of the channel thus allowing a greater prediction horizon and simpler predictor designs. The extracted parameters are then tracked over time and multipath gains are predicted using a linear model that is recursively updated. The prediction scheme is applied to the measured MIMO impulse responses to demonstrate the applicability of the method.

Keywords-fading prediction, channel parameter estimation, parameter tracking.
\end{abstract}

\section{INTRODUCTION}

Wireless systems are subject to fading - time variations of the receiving conditions caused by multipath propagation and transceiver movements. The received power undergoes deep fades within a time-frame corresponding to one wave-length which, for a typical communication system, amounts to movements on the centimeter scale. For efficient transmission, the communication system has to be able to mitigate fading effects. Should the current Channel State Information (CSI) be known in advance, the transceiver could reallocate internal resources in a better way or alter the transmission scheme in anticipation of the future conditions. This can be accomplished by predicting the CSI.

Fading mitigation by means of channel prediction has been studied and proved viable in a number of works [1]-[10]. These techniques were used to aid power control and resource allocation [3], [6], downlink diversity and adaptive modulation [1], [9]. It is often assumed that fading is a deterministic sinusoidal process with timevarying parameters that can be described using a discrete scatterer propagation model. The time-variation of the process parameters can then be modeled using linear (based on auto-regressive models) [2], [7] or nonlinear [3], [10] techniques. In the latter, the authors treat fading as dynamical process, producing the observed channel taps as the output. Predictions are then made by propagating the learned models into the future. These methods were studied for Single-InputSingle-Output (SISO) narrow-band [2], [4], [9], as well as for wideband channels [8]. In [6] it has been recently proposed to combine different channels in a smart-antenna system for prediction of the downlink received power. However, the authors only consider the narrowband case. In this contribution we discuss our preliminary results on predicting the Multiple-Input-Multiple-Output (MIMO) channels that undergo fast non-flat fading, i.e., the wideband case.
As compared to the SISO case, multiple antenna systems deliver much larger amounts of information with rich internal structure. Not exploiting this information might be too wasteful. Since fading results from the interaction between different multipath components, it can be advantageous to try to estimate individual multipaths using multiresolution techniques and treat each component as an individual channel. For example, in the Single-Input-Multiple-Output (SIMO) case, each multipath can be described by a multipath complex gain $a_{l}$, delay $\tau_{l}$, Doppler shift $\nu_{l}$, and Direction of Arrival (DoA) $\phi_{l}{ }^{1}$. This scheme can be generalized straight-forwardly to the wideband MIMO, as well as SISO and MISO systems.

In practical channels not only power (i.e., multipath gains) but also the other multipath parameters vary with time. This puts additional constraints on the predictor design. Thus, in this study we address the following questions:

- Is multipath-oriented prediction of channels viable?

- How to track time-varying channel and predictor parameters?

- How far can we predict with these models?

In the following sections we will subsequently answer those questions. Due to the space limitation, we will consider only SIMO channels for prediction since the presented approach can be easily extended to other channel configurations. Throughout the text we will demonstrate the performance of the proposed algorithms on the measured MIMO channels obtained by Forschungszentrum Telekommunikation Wien (FTW) in Vienna, Austria, under the supervision of Helmut Hofstetter [11]. The measurements were done with the MIMO capable wideband vector channel sounder RUSK-ATM, manufactured by MEDAV [12]. The sounder was specifically adapted to operate at the center frequency of $2 \mathrm{GHz}$. The transmitted signal was generated in the frequency domain to yield a predefined spectrum over $120 \mathrm{MHz}$ bandwidth with an approximately constant envelope over time. Two simultaneously multiplexed antenna arrays have been used at the transmitter and receiver. The transmitter was a uniform circular array with 15 sensors spaced at $\approx 6.45 \mathrm{~cm}$. The receiver was a fixed uniform linear array, with 8 sensors spaced half a wavelength apart, $\lambda / 2 \approx 7.5 \mathrm{~cm}$. The measurements were performed outdoors, with the transmitter array mounted on the roof of a building and the receiver moving with a velocity of $\approx 1 \mathrm{~m} / \mathrm{s}$. A MIMO channel snapshot was recorded every $20 \mathrm{msec}$, thus resulting in a spatial resolution of $\approx \lambda / 7$. For our purposes we will further select only a SIMO subset by taking a single transmitting antenna from the TX array.

The following sections are organized as follows: In Section II we introduce the multipath channel model we rely on; Section III introduces and explains the main steps in predictor design, and finally, Section IV shows some application results for real measured

${ }^{1}$ For simplicity, we will not account for the polarization and elevation angles. 


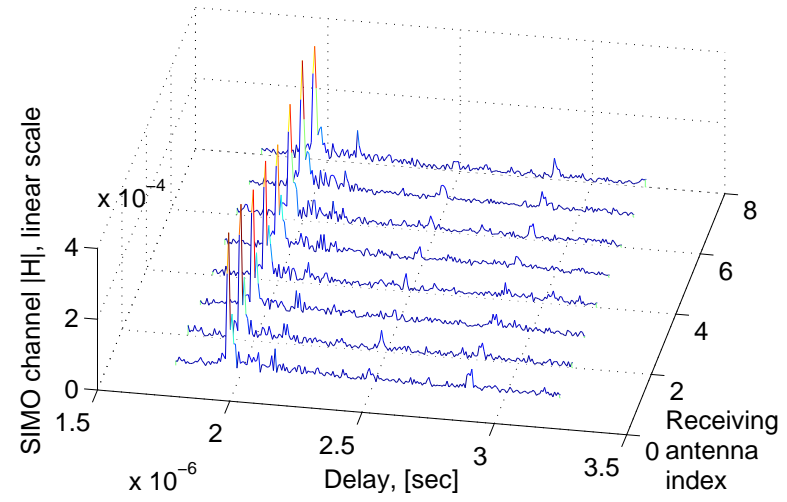

Fig. 1. A single snapshot of a SIMO impulse response.

channels.

\section{ChANNEL MODEL}

Let us assume that the receiver $(\mathrm{Rx})$ is equipped with an antenna array consisting of $P$ sensors located at $\boldsymbol{r}_{0}, \ldots, \boldsymbol{r}_{P-1} \in \mathbb{R}^{2}$ with respect to an arbitrary reference point. The corresponding equivalent baseband SIMO channel is modeled as

$$
\boldsymbol{h}_{t}(\tau)=\sum_{l=1}^{L} a_{l}(t) \boldsymbol{c}\left(\phi_{l}(t)\right) \mathrm{e}^{j \nu_{l}(t) t} \delta\left(\tau-\tau_{l}(t)\right),
$$

where $\boldsymbol{h}_{t}(\tau) \in \mathbb{C}^{P}$ is a vectorized representation of the time-varying SIMO channel impulse response, $a_{l}(t)$ and $\tau_{l}(t)$ are the gain and delay of the $l$ th multipath component at the time $t$, respectively. The phase term $\mathrm{e}^{j \nu_{l}(t) t}$ amounts for the phase-shift induced by the corresponding Doppler frequency $\nu_{l}(t)$. The $P$-dimensional vector $\boldsymbol{c}\left(\phi_{l}(t)\right)$ is known as the steering vector of the array and, provided the coupling between the antenna sensors can be neglected, it is represented as $\boldsymbol{c}\left(\phi_{l}(t)\right)=\left[c_{0}\left(\phi_{l}(t)\right), \ldots, c_{P-1}\left(\phi_{l}(t)\right)\right]^{T}$, where $\phi_{l}(t)$ is the Direction-of-Arrival of the $l$-th multipath ray. The components of $\boldsymbol{c}\left(\phi_{l}(t)\right)$ are given as

$$
c_{p}\left(\phi_{l}(t)\right)=f_{p}\left(\phi_{l}(t)\right) \exp \left(j 2 \pi \lambda^{-1}\left\langle\boldsymbol{e}\left(\phi_{l}(t)\right), \boldsymbol{r}_{p}\right\rangle\right)
$$

with $\lambda, e\left(\phi_{l}(t)\right)$, and $f_{p}\left(\phi_{l}(t)\right)$ denoting the wavelength, the unit vector in $\mathbb{R}^{2}$ pointing in the direction $\phi_{l}(t)$, and the complex electric field pattern of the $p$ th sensor, respectively. Channel model (1) can be used as a basis for different channel parameter estimation algorithms.

However, eq. (1) cannot be applied directly to the measured data. Practically, channel impulse responses (IR) are obtained using channel sounding that produces sampled representations of the channel impulse responses and include the influence of the sounder hardware. Thus, post-processing is needed to estimate the channel parameters from the measured data. An example of the measured SIMO impulse response is shown in Fig.1. SIMO channels are then represented as the consecutive snapshots of a complex matrix $\boldsymbol{H}[n] \in \mathbb{C}^{P \times M}, n=$ $0 \ldots N-1$, which is the sampled SIMO impulse response with rows corresponding to the antenna sensors and columns to the channel taps.

\section{CHANNEL PREDICTION}

We start by outlining our approach to channel prediction. The basic steps consist of:

- Parameter estimation: estimating the instantaneous values of the multipath parameters - multipath delays $\tau_{l}[n]$, Doppler frequencies $\nu_{l}[n]$, DoA's $\phi_{l}[n]$, and multipath gains $a_{l}[n], l=$ $1 \ldots L$, for $L$ multipath components.

- Parameter association: the obtained parameters should then be associated with the corresponding multipath trajectories to make the data tracks consistent over time.

- Predictor design and update: once the tracking has been solved, the parameter estimates are then used to update the channel predictor.

Below, we will provide a more detailed explanation of each of these steps.

\section{A. Parameter estimation}

Estimation of the multipath parameters $\left\{a_{l}, \tau_{l}, \nu_{l}, \phi_{l}\right\}$ from the measurement data has been extensively studied [13]. The most popular methodologies could be grouped into three major categories: spectral estimation (MUSIC), parametric subspace-based estimation (ESPRIT or unitary ESPRIT), and deterministic parametric estimation (SAGE) [14]. The latter is more suited for our application, since SAGE allows to jointly find the ML estimates of the multipath parameters and can be extended, if needed, to estimate other multipath parameters, like polarization and Direction-of-Departure in the MIMO case. For other methods the joint estimation might be computationally more expensive as compared to the SAGE. Unfortunately, the iterative nature of the SAGE algorithm requires a good initialization. However, in sequential processing, when SIMO channels arrive one after another, SAGE can be initialized using estimates obtained at the previous steps. Due to space limitations, the basic steps of the SAGE algorithm are not presented here and the interested reader will find a detailed algorithm description in [14].

The crucial point in wireless channel parameter estimation is the number of multipath components $L$. Generally, for real measurements, the number of multipath components may vary with time. This presents a substantial difficulty not only for parameter estimation algorithms, but also for parameter tracking. The number of multipaths can be estimated 'on-line', if desired, using information-theoretic criteria [15]. Accommodation of this situation goes beyond the scope of the presented work. To account for it we will simply fix $L$ to a number large enough to capture the most dominant waves.

In Figure 2 we present the scatter plot of the estimated multipath parameters for the time-varying SIMO channels. To generate the plot, a block of 5 consecutive SIMO channel snapshots was used to estimate $L=20$ dominant multipath components with the corresponding parameters. Assuming a velocity of $1 \mathrm{~m} / \mathrm{s}$ the timeaxis in Fig. 2 has been re-scaled in wave-lengths to demonstrate the spatial channel variation.

Unfortunately, parameter estimation algorithms do not provide any ordering information on how to associate the multipath components at time $n-1$ with those at time $n$. In order to be able to learn the parameter dynamics for prediction purposes, it is necessary to reconstruct this ordering, i.e., to associate the consecutive parameter estimates that correspond to the same physical multipath component over time.

\section{B. Parameter tracking}

In general, parameter tracking/association is not a trivial problem since there is no a priory model that can be used to ease this task. However, this model can be constructed iteratively, as the algorithm proceeds. Indeed, the sought predictor is the needed model of the track dynamics. Our solution lies in coupling prediction with the dynamic programming techniques for searching the optimal parameter associations. 


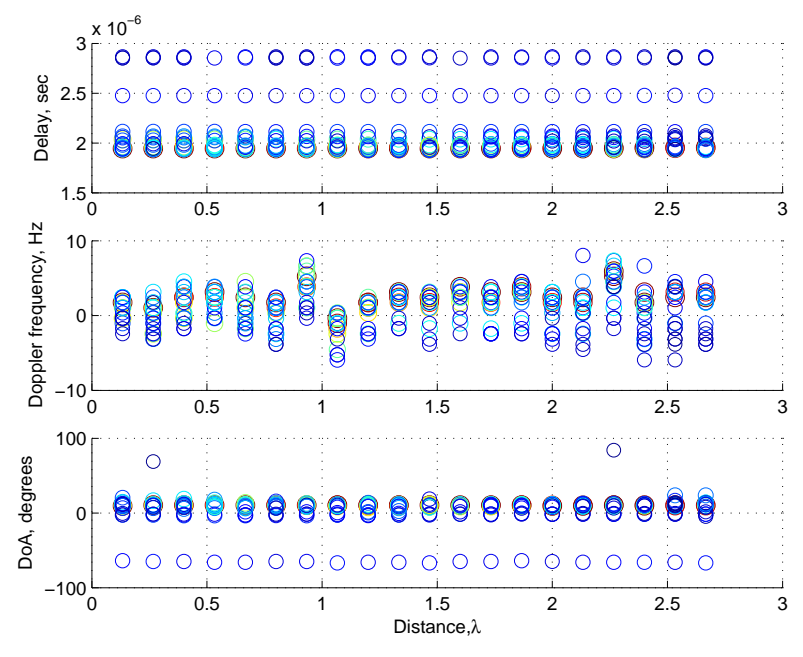

Fig. 2. Scatter plot of the estimated parameters.

For tracking purposes it might be advantageous to consider only a subset of all the estimated multipath components. The difficulty in parameter tracking is that some tracks may cease to exist, while others are born. Instead of introducing the 'death-birth' process to model these situations, we settle for such tracks that will not disappear within the observation window, or if they do, the effect on the predictor will be minimal. Basically, this would correspond to reconstructing $K \leq L$ strongest multipath components, each represented by a time-varying set of parameters

$$
\boldsymbol{\theta}_{k}[n]=\left\{\tau_{k}[n], \nu_{k}[n], \phi_{k}[n], a_{k}[n]\right\}, k=1 \ldots K .
$$

Let us assume for the moment that the dynamics of each track is captured by a certain known deterministic hypermodel $\boldsymbol{H}_{k}(\cdot)$, in a sense that

$$
\boldsymbol{\theta}_{k}[n+1]=\boldsymbol{H}_{k}\left(\boldsymbol{\theta}_{k}[n], \boldsymbol{\theta}_{k}[n-1], \ldots\right) .
$$

The term hypermodel is used to stress that $\boldsymbol{H}_{k}(\cdot)$ encodes the dynamics of the 'underlying' channel. Later we will show how this model can be learned.

For tracking purposes not all of the multipath parameters contribute equally to deciding between several alternative track continuations. Thus, only a subset of the available parameters, namely multipath delay, Doppler shift, and DoA ${ }^{2}$ might be used. Let $\boldsymbol{m}_{l}[n] \in \boldsymbol{\theta}_{l}[n], l=$ $1 \ldots L$, and $\boldsymbol{t}_{k}[n] \in \boldsymbol{\theta}_{k}[n], k=1 \ldots K$, denote a subset of parameters (we will call them centers) that will be used for tracking, i.e. $\boldsymbol{t}_{k}[n]=\left\{\tau_{k}[n], \nu_{k}[n], \phi_{k}[n]\right\}$ and $\boldsymbol{m}_{l}[n]=\left\{\tau_{l}[n], \nu_{l}[n], \phi_{l}[n]\right\}$. Now, at the time instance $n$ the estimation algorithm generates a new set of parameters $\left\{\boldsymbol{\theta}_{l}[n]\right\}_{\tilde{l}=1}^{L}$. The hypermodels $\boldsymbol{H}_{k}$ can be then used to generate predictions $\tilde{\boldsymbol{\theta}}_{k}[n]=\boldsymbol{H}_{k}\left(\boldsymbol{\theta}_{k}[n-1], \boldsymbol{\theta}_{k}[n-2], \ldots\right)$ for the $K$ tracks of interest. The corresponding centers $\boldsymbol{m}_{l}[n]$ and $\tilde{\boldsymbol{t}}_{k}[n] \in \tilde{\boldsymbol{\theta}}_{k}[n]$ can be obtained by selecting the subsets of the corresponding parameter sets. Then the data association can be cast as the standard Linear Programming problem. Consider the graph in Fig. 3. Each edge that connects two centers $\tilde{\boldsymbol{t}}_{k}[n]$ and $\boldsymbol{m}_{l}[n]$ induces a cost

$$
C_{k l}[n]=f\left(\tilde{\boldsymbol{t}}_{k}[n], \boldsymbol{m}_{l}[n]\right)+\mu C_{k}[n-1] .
$$

\footnotetext{
${ }^{2}$ In the SIMO case, multipath delay, Doppler shift and DoA fully determine the multipath component, and thus should be used for tracking. Although the multipath amplitude(gain) is also estimated, it does not help to distinguish two different components.
}

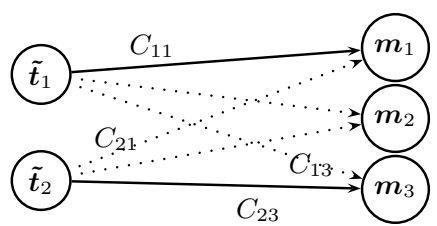

Fig. 3. Possible track continuations for $K=2$ and $L=3$

Here $C_{k}[n-1]$ is the cost accumulated by the $k$ th track at the time $n-1$, and $0 \leq \mu \leq 1$ is a forgetting factor. Now, let us define a binary variable $x_{k l}$ such that:

$$
x_{k l}=\left\{\begin{array}{lc}
1, & \text { if } \boldsymbol{m}_{l}[n] \text { should be assigned to } \tilde{\boldsymbol{t}}_{k}[n] \\
0, & \text { otherwise }
\end{array}\right.
$$

Then, the optimal track selection should minimize the total induced $\cos t Z$ :

$$
\begin{aligned}
& \underset{x_{k l}}{\operatorname{argmin}} Z=\sum_{k=1}^{K} \sum_{l=1}^{L} C_{k l}[n] x_{k l}, \quad \text { so that } \\
& \sum_{l=1}^{L} x_{k l}=1, k=1 \ldots K, \quad \text { and } \quad x_{k l} \in\{0,1\} .
\end{aligned}
$$

Should we have just a single track, i.e., $K=1$, the optimal solution could be found using the Viterbi algorithm. However, in our case all of the $K$ tracks have to be simultaneously associated with $L$ candidates, which makes the problem more difficult. Problem (2) can be solved using standard linear programming techniques (see, for example, [16]). In our experiments the cost function $f(\cdot, \cdot)$ is selected as a weighted ${ }^{3}$ Euclidean distance, however more sophisticated costs can be imaged. In all the experiments the forgetting factor was set to $\mu=0.995$.

\section{Hypermodel learning and parameter prediction}

In our case, the hypermodels $\boldsymbol{H}_{k}$ are used not only to make predictions of the channel components, but also as a part of the data tracking/association scheme. This makes both very dependent on each other: proper parameter tracks are needed to learn the 'correct' hypermodel, and a correct hypermodel is needed to solve the data association problem. Since the data (multipath parameter estimates) arrive sequentially, it makes sense to adopt Bayesian sequential methods for building predictors. The individual multipath components supposedly have simpler dynamics than the full SIMO channel. Thus predictors based on linear models can be sufficiently accurate.

As our experiments show, the delays, Doppler frequencies, and DoA's trajectories can be well represented by simple local linear trends. Amplitudes however vary much stronger, thus requiring more elaborate models. We propose to use two different Kalman filters $(\mathrm{KF})$ for parameters tracking and prediction for each track. The corresponding filter structures are described below.

1) Tracking of the delay, Doppler frequency and DoA: The tracking of these parameters can be accomplished with a simple damped linear trend KF [17]. For a single track, the state-space representation of this filter for the delay tracking is given as (we will skip the

${ }^{3}$ Since delay, Doppler and DoA components in the vector usually take values that might differ by several orders of magnitude, appropriate weighting is necessary to make sure that each component contributes accordingly to the computation of the cost. 
subscript $(\cdot)_{k}$ to simplify the notations):

$$
\begin{aligned}
& {\left[\begin{array}{c}
\hat{\tau}[n+1] \\
v_{\tau}[n+1]
\end{array}\right]=\left[\begin{array}{cc}
1 & 1 \\
0 & \delta_{\tau}
\end{array}\right]\left[\begin{array}{c}
\hat{\tau}[n] \\
v_{\tau}[n]
\end{array}\right]+\boldsymbol{\xi}_{\tau}[n],} \\
& \tau[n]=\left[\begin{array}{ll}
1 & 0
\end{array}\right]\left[\begin{array}{c}
\hat{\tau}[n] \\
v_{\tau}[n]
\end{array}\right]+\epsilon_{\tau}[n] .
\end{aligned}
$$

In (3), $\hat{\tau}[n]$ is the smoothed estimate of the multipath delay $\tau[n]$ and $v_{\tau}[n]$ is the slope of the linear trend. The coefficient $\delta_{\tau} \leq 1$ is a damping factor of the trend extrapolator. It is has been shown [17] that for an $i$-step ahead predictor based on the information up to the moment of time $n$, (3) will converge to the value $\hat{\tau}=\tau[n]+$ $v_{\tau}[n] /\left(1-\delta_{\tau}\right)$ as $i \rightarrow \infty$. Equations (3) can be easily augmented to include the Doppler frequency and the DoA components in order to allow simultaneous tracking of the components of $\boldsymbol{t}_{k}[n]$.

Although our model is assumed to be deterministic, the actual observations are not since in reality multipath parameters cannot be estimated with zero variance. Since parameter estimates provided by SAGE are unbiased and consistent [14], the disturbances $\epsilon_{\tau}[n]$ can be thought as white Gaussian estimation noise. State noise $\boldsymbol{\xi}_{\tau}[n]$ is chosen to be close to zero since we assume a deterministic model for the parameter variation.

2) Tracking amplitudes: In power prediction we are mostly interested in accurately predicting the evolution of the amplitudes since they capture how much power a multipath component possesses. In order to achieve this goal more complicated predictors are required. In this paper, we will utilize an adaptive linear predictor in the following form:

$$
\hat{a}_{k}[n+\mathcal{L}]=\sum_{i=0}^{Q-1} w_{i}[n] a_{k}[n-i]=\boldsymbol{w}_{k}[n]^{T} \boldsymbol{a}_{k}[n]
$$

where $\mathcal{L} \geq 1$ is the prediction horizon, and $Q>0$ is the order of the predictor. We also defined $\boldsymbol{a}_{k}[n]=\left[a_{k}[n], \ldots, a_{k}[n-Q+1]\right]^{T}$ and $\boldsymbol{w}_{k}[n]=\left[w_{0}[n], \ldots, w_{Q-1}[n]\right]^{T}$. Estimation of the predictor coefficients $\boldsymbol{w}_{k}[n]$ can be done using standard adaptive algorithm. Here we propose to use two adaptive schemes to learn the predictor coefficients. The first one is based on the standard Recursive Least Squares (RLS) algorithm [18]. The second one, similarly to the tracking of the delays and the other parameters, utilizes the KF framework. For $\mathcal{L}=1$ we can represent the predictor in state-space form as follows:

$$
\left\{\begin{aligned}
{\left[\begin{array}{c}
\hat{\boldsymbol{a}}_{k}[n+1] \\
\boldsymbol{w}_{k}[n+1]
\end{array}\right] } & =\left[\begin{array}{cc}
\boldsymbol{w}_{k}[n]^{T} & \mathbf{0} \\
\mathbf{0} & \boldsymbol{I}
\end{array}\right]\left[\begin{array}{c}
\hat{\boldsymbol{a}}_{k}[n] \\
\boldsymbol{w}_{k}[n]
\end{array}\right]+\boldsymbol{\eta}_{k}[n] . \\
a_{k}[n] & =\left[\begin{array}{ll}
1 & \mathbf{0}
\end{array}\right]\left[\begin{array}{l}
\hat{\boldsymbol{a}}_{k}[n] \\
\boldsymbol{w}_{k}[n]
\end{array}\right]+\varsigma_{k}[n] .
\end{aligned}\right.
$$

This formulation is also known as the Joint Extended Kalman Filter (EKF). It can be seen that it forms a bilinear state-space representation and this necessitates the usage of the EKF (see [19, sec. 5] for more details on Joint EKFs). Similarly to (3), $\varsigma_{k}[n]$ plays the role of the observation noise and state noise $\boldsymbol{\eta}_{k}[n]$ is set close to zero.

The substantial distinction between these two approaches is that RLS-learned predictor is trained for a particular prediction horizon $\mathcal{L}$, while in case of the EKF-learned predictor the forecasts for $\mathcal{L}>1$ are obtained by recursive application of (5) $\mathcal{L}$ times.

\section{SOME PREDICTION RESULTS FOR MEASURED CHANNELS}

Now, we have all the ingredients to perform prediction of the measured data. Assuming the data has been sampled at the Nyquist rate, we upsample the obtained channels by the factor of 5 . This is done to ease the parameter association/tracking step. Then, for each block of 5 SIMO channel snapshots, $L=20$ parameters were
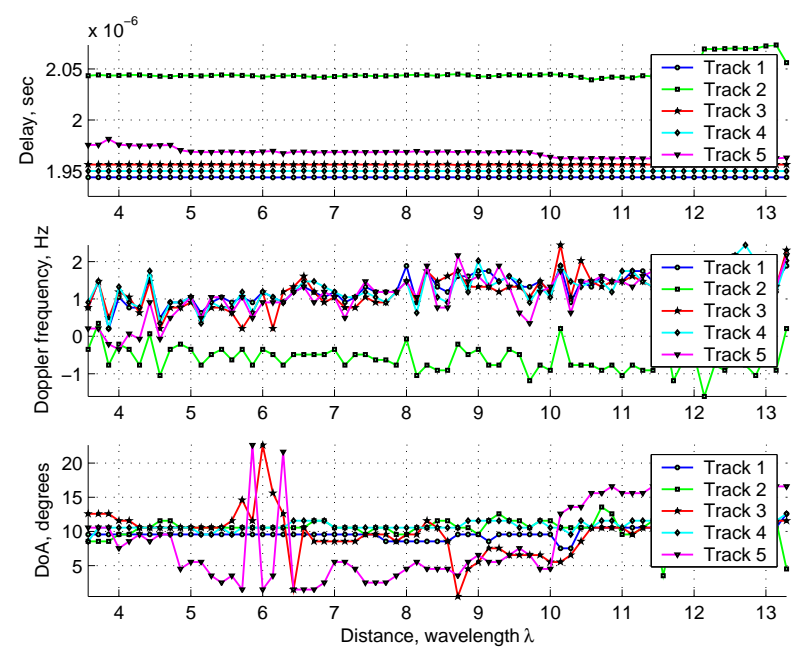

Fig. 4. Tree reconstructed tracks.

estimated and $K=5$ strongest components were followed over time. Figure 4 shows the reconstructed tracks for a time-varying situation when the mobile moves over a distance of $\approx 10$ wavelengths ${ }^{4}$. For both predictors the filter order was set $Q=3$ samples.

In order to evaluate the prediction performance, we introduce the notion of the naive predictor. If our RLS- or EKF-learned predictor is based on (4), the naive predictor in both cases assumes that $\hat{a}_{k}[n+\mathcal{L}]=\hat{a}_{k}[n]$, i.e., this is a predictor of order $Q=0$ that makes predictions by simply keeping the last estimated state value in case of EKF-learned, or last seen value in case of RLS-learned predictor, respectively. The naive and true learned predictors can be compared by analyzing the corresponding prediction errors $e_{\text {naive }}[n]$, $e_{\mathrm{rls}}[n]$, and $e_{\mathrm{ekf}}[n]$. Using these prediction errors, we can define the prediction gain (PG) $G_{\text {pred }}$ as follows

$$
\begin{aligned}
G_{\text {pred }}^{\mathrm{RLS}} & =10 \log _{10}\left(\frac{\sigma_{\text {true }}^{2}}{\sigma_{\text {rls }}^{2}}\right), \\
G_{\text {pred }}^{\mathrm{EKF}} & =10 \log _{10}\left(\frac{\sigma_{\text {true }}^{2}}{\sigma_{\text {ekf }}^{2}}\right), \\
G_{\text {pred }}^{\mathrm{NAIVE}} & =10 \log _{10}\left(\frac{\sigma_{\text {true }}^{2}}{\sigma_{\text {naive }}^{2}}\right),
\end{aligned}
$$

where $\sigma_{\text {true }}^{2}$ is the variance of the true signal that is to be predicted, and $\sigma_{\text {naive }}^{2}, \sigma_{\text {rls }}^{2}$, and $\sigma_{\text {ekf }}^{2}$ are the variance of the prediction error for the naive, RLS-, and EKF-learned predictors, respectively. It should be noted, that the prediction error is generally non-stationary due to the possible non-stationary behavior of the reconstructed tracks. This would make the prediction error to be good for some segments, but worse if the predictor has to re-learn the data. To accommodate this situation we segment the error signals into chunks equivalent to $\approx 4 \lambda$ and compute $(6)$ for each individual segment. The obtained results are then averaged over the whole data sequence. In Figure 5 one can see the performance of the proposed predictors for different prediction horizons. We plotted the PG's for the strongest multipath component, as well as those averaged over the other components. It can be seen that with the RLS-learned predictor it is possible to achieve prediction horizons with positive prediction gains up to $3 \lambda$. As compared to [6] where the achievable prediction horizons of $\approx 1.6 \lambda$ were reported for the simulated data, and $\lambda / 60$ for the

${ }^{4}$ The distance of $10 \lambda$ corresponds to the physical distance of $1.5 \mathrm{~m}$ 


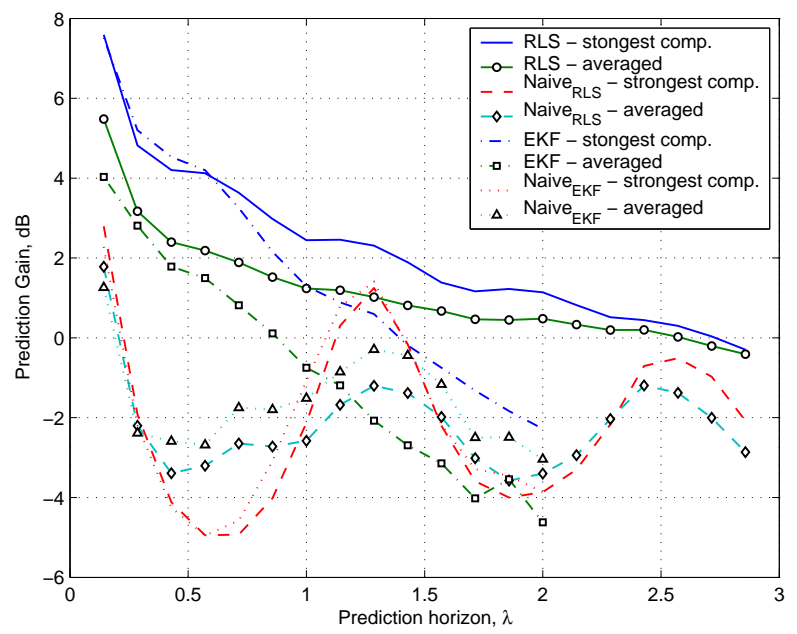

Fig. 5. Prediction gain for different prediction horizons.

measured IR with the spatial resolution of $\lambda / 600$, prediction horizons of almost $3 \lambda$ for the measured channels in our case is a significant improvement. It should be noted, that in our case the spatial resolution is much lower, i.e., only 7 samples per wavelength, however the achieved prediction horizons for the real channel IR's are longer. We also see that for short prediction horizons both predictors give similar results. However, the iterated EKF-based predictor is not able to forecast far into the future. Shortening of the prediction horizon in this case is due to the stability issues that arise when (5) is iterated many times.

We also observed that increasing the length of the predictor, i.e. using $Q>3$, does not significantly improve the prediction performance. This fact supports our assumption that the multipathbased approach to channel prediction reduces the complexity of the required predictor, which is an advantage if such a system is to be implemented in a hardware.

\section{CONCLusions}

In this work we presented an algorithm for predicting fading in wireless communication channels. Unlike previous works where the taps of the sampled channel IR were used to train predictors, we decided to first extract the individual multipath components, thus alleviating the fading effects at the first place. The forecasts are then done for each extracted multipath. This significantly decreases the complexity of the resulting predictors and extends the prediction horizons. Although this approach requires proper tracking to reconstruct the multipath dynamics, it allows to predict the channel parameters as far as three wavelengths into the future, which would correspond to almost half a meter distance in our case, even for low spatial channel sampling. It should be stressed, however, that dense spacial sampling is important for proper parameter association and tracks reconstruction. The obtained prediction results prove that the new method can be considered for channel prediction, however more elaborate comparison with other methods in terms of overall predictor complexity is still needed. The proposed method can also be extended to wideband MIMO, as well as SISO and MISO channels, and it can be used not only to predict the power variations of the multipath components, but also to predict the full structure of the impulse response. This substantially widens the applicability of the proposed algorithm.

\section{REFERENCES}

[1] H. Duel-Hallen, A. Shengquan Hu Hallen, "Long-range prediction of fading signals," IEEE Signal Processing Magazine, vol. 17, no. 3, pp. 62 - 75, May 2000.

[2] J.K. Hwang and J.H. Winters, "Sinusoidal modeling and prediction of fast fading processes.," in Global Telecom. Conf., GLOBECOM'98. IEEE, 1998, vol. 2, pp. 892-896.

[3] T. Ekman, Prediction of Mobile Radio Channels: Modeling and Design, Ph.D. thesis, Signal Processing at Uppsala University, Nov. 2002.

[4] Eyceoz T., Duel-Hallen A., and Hallen H., "Deterministic channel modeling and long range prediction of fast fading mobile radio channels," IEEE Communications Letters, vol. 2, no. 9, pp. 254-256, Sep. 1998.

[5] Andersen J.B., Jensen J., Jensen S.H., and Frederiksen F., "Prediction of future fading based on past measurements," in 50th IEEE Conf. on Vehic. Tech.,VTC'99, 1999, vol. 1, pp. 151 - 155.

[6] K.R. Guanghan Xu Arredondo, A. Dandekar, "Vector channel modeling and prediction for the improvement of downlink received power," IEEE Trans. on Comm., vol. 50, no. 7, pp. 1121-1129, Jul 2002.

[7] R. Vaughan, P. Teal, and R. Raich, "Short-term mobile channel prediction using discrete scatterer propagation model and subspace signal processing algorithms," in 52nd IEEE Conf. on Vehic. Tech., 2000, vol. 2, pp. $751-758$.

[8] Liang Dong, Guanghan $\mathrm{Xu}$, and Hao Ling, "Prediction of fast fading mobile radio channels in wideband communication systems," in IEEE Global Telec. Conf., GLOBECOM '01., Nov 2001, vol. 6, pp. 3287 3291.

[9] S. Hu, H. Hallen, and A. Duel-Hallen, "Physical channel modeling, adaptive prediction and transmitterdiversity for flat fading mobile channel," in IEEE Workshop on Signal Proc. Advances in Wireless Comm. SPAWC, 1999, pp. 387-390.

[10] G. Ekman, T. Kubin, "Nonlinear prediction of mobile radio channels: measurements andmars model designs," in Proceedings of the IEEE Int. Conf. on Acoust., Speech, and Signal Proc., 1999, vol. 5, pp. 2667-2670.

[11] E. Bonek et al., "Double-directional superresolution radio channel measurements," Proc. Conf. on Comm., Control, and Computing, vol. 3, Oct. 2001.

[12] R. Thoma et al., "Identification of time-variant directional mobile radio channels," IEEE Trans. on Instrumen. and Meas., vol. 49:2, pp. 357-364, Apr. 2000.

[13] H. Krim and M. Viberg, "Two decades of the array signal processing research,” IEEE Signal Processing Mag., p. 6794, July 1996.

[14] B.H. Fleury, M. Tschudin, R. Heddergott, D. Dahlhaus, and K. Ingeman Pedersen, "Channel parameter estimation in mobile radio environments using the SAGE algorithm," IEEE Journal on Selected Areas in Comm., vol. 17, no. 3, pp. 434-450, March 1999.

[15] T. Wax, M. Kailath, "Detection of signals by information theoretic criteria," IEEE Trans. on Acoustics, Speech, and Signal Proc., vol. 33, no. 2, pp. 387-392, April 1985.

[16] H.A. Taha, Operations Research, Prentice Hall International, 2002.

[17] Andrew C. Harvey, Forecasting, structural time series models and the Kalman filter, Cambridge University Press., 1989.

[18] Haykin S., Adaptive Filter Theory, Prentice Hall, New Jersey, 1991.

[19] Haykin S., Ed., Kalman Filtering and Neural Networks, John Wiley \& Sons, Inc., 2001. 